\title{
Annie Crépin, Révolution et armée nouvelle en Seine- et-Marne (1791-1797)
}

\section{Philippe Catros}

\section{(2) OpenEdition \\ 12 Journals}

\section{Édition électronique}

URL : https://journals.openedition.org/ahrf/10669

DOI : 10.4000/ahrf.10669

ISSN : 1952-403X

Éditeur :

Armand Colin, Société des études robespierristes

\section{Édition imprimée}

Date de publication : 1 septembre 2009

Pagination : 201-203

ISBN : 978-2-200-92559-8

ISSN : 0003-4436

Référence électronique

Philippe Catros, «Annie Crépin, Révolution et armée nouvelle en Seine-et-Marne (1791-1797) 》, Annales historiques de la Révolution française [En ligne], 357 | juillet-septembre 2009, mis en ligne le 09

décembre 2009, consulté le 23 avril 2022. URL : http://journals.openedition.org/ahrf/10669 ; DOI : https://doi.org/10.4000/ahrf.10669

Ce document a été généré automatiquement le 23 avril 2022.

Tous droits réservés 


\title{
Annie Crépin, Révolution et armée nouvelle en Seine-et-Marne (1791-1797)
}

\author{
Philippe Catros
}

\section{RÉFÉRENCE}

Annie Crépin, Révolution et armée nouvelle en Seine-et-Marne (1791-1797), Paris, Éditions du Comité des travaux historiques et scientifiques, 2008, 463 p., ISBN 978-2-7355-0660-6, $32 €$

1 À rebours de ses grandes synthèses nationales comme La conscription en débat et Défendre la France, dans ce dernier ouvrage, Annie Crépin analyse la façon dont les levées d'hommes et la formation d'une armée nationale de citoyens-soldats ont été acceptées par la population du département de la Seine-et-Marne. La démarche est intéressante et mérite d'être saluée : Annie Crépin reprend en fait une partie de sa thèse de doctorat en l'enrichissant des acquis de ses travaux ultérieurs ainsi que des développements récents de la recherche historique sur la question militaire sous la Révolution.

2 La période étudiée va de 1791 à 1797, c'est-à-dire des premières levées d'hommes à la veille de l'instauration de la conscription militaire (loi Jourdan-Delbrel du 5 septembre 1798). L'ouvrage est divisé en quatre parties : la première est consacrée à la levée des volontaires nationaux de 1791, la deuxième aux levées de 1792, la troisième à celles de 1793, et la quatrième, enfin, aborde la question de l'effort de guerre pendant la Convention thermidorienne et le Directoire.

3 Exploitant la richesse des sources départementales et municipales, Annie Crépin ne limite pas son propos aux analyses quantitatives des levées révolutionnaires : chacune est contextualisée; et le rôle des autorités locales et départementales, voire des représentants du pouvoir central, dans le déroulement de chaque levée est mis en lumière; de même, les réactions des appelés, et plus généralement, de la société dans son ensemble sont abondamment décrites et illustrées. 
4 Le choix de la Seine-et-Marne relève de la volonté d'étudier les levées révolutionnaires dans « un département qui se rapprochait de la moyenne nationale ». Modéré, puisqu'il envoya des hommes de la Plaine à la Convention et qu'il «ne se signala ni par une hostilité, ni par un enthousiasme débordant à l'égard de la Révolution », il correspond parfaitement au portrait de la «France de l'ordre des plaines de grandes cultures » dressé par Michel Vovelle.

5 Les discours patriotiques enflammés des administrateurs et des commissaires aux différentes levées - exaltant la lutte défensive contre les tyrans plus que l'esprit de conquête ou le thème de la croisade révolutionnaire - ne doivent pas faire illusion. Ils ne reflètent que l'opinion d'une minorité de militants politiques ; pas l'esprit public d'un département étranger à tout nationalisme belliqueux. Cette atonie relative se remarque dès 1791, puisque, contrairement à d'autres départements, la population du département de la Seine-et-Marne ne forma pas spontanément de bataillons de volontaires nationaux - et il en fut de même lors de la levée des Fédérés l'année suivante.

6 Néanmoins, les levées de 1792 et 1793 ne soulevèrent pas de véritable opposition. Comme le remarque Annie Crépin, «les troubles, quand ils se manifestent, sont sporadiques et ne sont pas le fait des mêmes cantons d'une levée à une autre ». Il est de même difficile de voir dans ces résistances collectives une manifestation d'un comportement anti-révolutionnaire profondément marqué. À l'inverse, comme le montre l'exemple des cantons du Gâtinais qui, bien qu'ayant été le théâtre d'émeutes anti-seigneuriales au début de la Révolution, n'ont pas fait preuve d'un excès de zèle patriotique lors des levées ultérieures, le rejet de l'Ancien Régime ne débouche pas sur un enthousiasme révolutionnaire, ni «sur une prise de conscience plus large des intérêts de la Révolution et de la patrie menacée ».

7 Quant à l'analyse sociologique du recrutement, elle nous apprend que dès 1791 , les classes les plus modestes ont une propension plus grande que les couches plus aisées à rejoindre les drapeaux. La relative autonomie laissée aux administrations locales, mais aussi aux populations, pour fournir les contingents exigés, du moins jusqu'à la levée en masse, explique ce phénomène. Non seulement le remplacement individuel permet aux familles les plus aisées de trouver moyennant finance un substituant issu des catégories sociales inférieures, mais la pratique du remplacement collectif - c'est-à-dire que la population des cantons s'entendait pour fournir son contingent, quitte à payer les "volontaires »- accentue cette tendance. Ce ne sont pas seulement les bourgeois qui échappent ainsi à l'impôt du sang, mais aussi les fils de petits propriétaires paysans.

8 Annie Crépin n'en conclut pas - à juste titre - que cette attitude traduit uniquement un manque de patriotisme de leur part. Dans des sociétés rurales encore profondément marquées par les pratiques communautaires, ces comportements révèlent que les levées sont avant tout conçues comme des contributions collectives. Doivent partir à l'armée les jeunes les moins nécessaires à la survie de la communauté ; les hiérarchies locales, les réseaux de pouvoir et le clientélisme jouant ainsi un rôle prépondérant dans la désignation de nombreuses recrues. Est-ce pour autant le signe d'une résistance aux levées? Les communautés rurales ne le perçoivent pas forcément ainsi. Alors que l'obligation militaire est appréhendée plus comme une contribution collective que comme un devoir personnel, elles estiment avoir répondu à l'appel des autorités constituées en répartissant la charge de l'obligation entre ceux qui contribuent en personne et ceux qui contribuent financièrement à habiller et à équiper un soldat - ce 
que les levées exigent -, voire à lui octroyer un petit pécule, ou encore à aider sa famille.

Certes cette pratique du recrutement contredit l'égalité révolutionnaire - qu'y-a-t-il de commun, en effet, entre un jeune homme qui quitte sa communauté pour risquer sa vie à la guerre et ceux qui estiment avoir accompli leurs obligations envers l'État par une contribution pécuniaire? Mais à ne vouloir aborder les attitudes face au recrutement que sous l'angle de la conception jacobine de l'égalité entre individus semblables, on passe sans doute à côté de la réalité complexe d'un monde rural encastré dans des pratiques communautaires bien éloignées des modèles d'une élite politique éclairée imprégnée de la pensée des Lumières.

10 La levée en masse - avant la grande loi de conscription de l'an VI-, en imposant un service militaire personnel aux jeunes gens, fera triompher, en façade du moins, la conception jacobine de l'obligation militaire. La population de la Seine-et-Marne s'y pliera sans guère résister ; les enfants des petits propriétaires paysans venant rejoindre sous les drapeaux ceux des levées précédentes, achevant ainsi la formation d'« une armée citoyenne, égalitaire tant dans son mode de recrutement que dans sa structure interne ».

11 C'est bien là une des conclusions les plus importantes du travail d'Annie Crépin. L'armée nationale de la Révolution n'est pas seulement née de la levée en masse. L'exemple du département de la Seine-et-Marne montre que la démocratisation du recrutement se fi t dès 1791 et que les bases sociologiques de l'armée des soldats de l'an II y ont donc été jetées dès les premières levées révolutionnaires. Cette conclusion rend donc caduque, à l'échelle de la Seine-et-Marne, l'interprétation classique opposant la levée «bourgeoise » des volontaires nationaux de 1791 aux «levées populaires » de 1792 et 1793. 